\title{
Cambios físicos en estudiantes del programa de cultura física, deporte y recreación (2013-2016)
}

\author{
Laura Elizabeth Castro Jiménez" ${ }^{1}$ Yenny Paola Argüello Gutiérrez², \\ Oscar David Godoy Gómez $z^{3}$ Daiver Steven Sabogal Romero ${ }^{4}$
}

\section{Resumen}

Objetivo: determinar los cambios de los estudiantes de seis cohortes (2013-1, 2013-2, 2014-1, 2014-2, 2015-1, 2015-2), evaluados al ingreso de la universidad, y compararlos con los resultados a corte 2017-I en la aptitud física evaluada a través de la composición corporal, fuerza, flexibilidad, velocidad, agilidad y consumo de oxígeno. Metodología: estudio de corte cuantitativo, de alcance descriptivo, con una cohorte de seguimiento prospectivo desde el 2013-I hasta el 2016-II, con corte a 2017-I. Los criterios de inclusión fueron hombres y mujeres, estudiantes de la Facultad de Cultura Física, Deporte y Recreación de la Universidad Santo Tomás, que hayan permanecido activos como estudiantes desde el ingreso hasta la fecha del corte que es 2017-I. Resultados: después de comparar los resultados de ingreso frente a los resultados a corte 2017-1, se encontró una mejora en la prueba 30 metros lanzados, adaptación al móvil (agilidad) y los valores generales de fuerza (espalda y mano), así como una disminución en las pruebas de agilidad (obstáculos) y flexibilidad. Conclusión: los cambios que se dan en los estudiantes en su condición física en su paso por la universidad no siempre implican mejora; por lo evidenciado, se encuentra disminución de algunas cualidades del movimiento, lo que no se puede atribuir completamente a los procesos de envejecimiento, mostrando que el paso por la universidad y lo que implica la permanencia en ella genera conductas que repercuten a este resultado.

Palabras clave: aptitud física; criterios de admisión escolar; estudiantes.

1 Fisioterapeuta. Magister en Salud Pública. Doctorando en Humanidades, Humanismo y Persona. Docente Investigadora Grupo Cuerpo, Sujeto y Educación. Universidad Santo Tomás. Contacto: laura.castro@usantotomas.edu.co. Orcid: https://orcid.org/0000-0001-5166-8084

2 Fisioterapeuta. Magister en Fisiología. Docente Investigadora Grupo Cuerpo, Sujeto y Educación. Universidad Santo Tomás. Contacto: yenniarguello@usantotomas.edu.co. Orcid: https://orcid. org/0000-0001-8335-4936

3 Profesional en Cultura Física, Deporte y Recreación. Grupo Cuerpo, Sujeto y Educación. Universidad Santo Tomás. Contacto: oscar.godoy@usantotomas.edu.co. Orcid: https://orcid.org/0000-0002$1955-2388$

4 Profesional en Cultura Física, Deporte y Recreación. Grupo Cuerpo, Sujeto y Educación. Universidad Santo Tomás. Contacto: daiver.sabogal@usantotomas.edu.co. Orcid: https://orcid.org/0000-00026173-9189 


\section{Introducción}

A nivel mundial, las universidades que ofertan los programas de Educación Física y áreas afines poseen pruebas de admisión que valoran, en su gran mayoría, capacidades físicas, condicionales y coordinativas, con el fin de aceptar a los estudiantes que posean las mejores cualidades físicas desde el ingreso y que se puedan potencializar a lo largo de la carrera, para de esta manera tener egresados con condiciones excepcionales en el medio laboral. La Facultad de Cultura Física, Deporte y Recreación posee un proceso de admisiones que busca evaluar la competencia cinética corporal, las competencias básicas y el contexto sociocultural de los jóvenes interesados en ingresar al programa de pregrado de la Facultad, evaluando desde su integralidad a los aspirantes, para seguir con esta formación a lo largo de la carrera.

El programa reconoce que cada joven requiere una formación cognitiva, de conocimientos especializados y de aspectos que lo formen como una persona ética y con sentido humanista. Por ello, es importante identificar en los jóvenes su inclinación por la práctica de actividad física, el deporte y su interés por los hábitos de vida saludable, por ello "el estudiante debe poseer un nivel de acondicionamiento físico, demostrar cierto grado de competencias básicas y unos niveles de desarrollo personal" (Córdoba, 2013, p. 6), con el fin de que durante la carrera mejore las condiciones de base o las mantenga, para que exista coherencia entre lo que se dicta y lo que se hace. Así pues, para implementar las pruebas de admisión en la Facultad se lleva a cabo un proceso de diseño, pilotaje y validación que se llevó a cabo por primera vez desde el año 2011 al año 2012, luego de este periodo se inicia, a partir del segundo semestre del año 2013, la aplicación de las diferentes pruebas de ingreso.

Durante los últimos 4 años las pruebas han sido aplicadas, pero aún no se ha realizado ningún proceso de seguimiento ni investigación que permita identificar claramente el perfil de los estudiantes que ingresan a la Facultad y los que terminan la carrera, tampoco se conoce con claridad el comportamiento de la condición física a lo largo del tiempo, es decir, no se reconoce desde su ingreso hasta el semestre actual que cursa qué cambios existen en la condición física de cada estudiante, si efectivamente mejoró — que es el objetivo de la 
formación de la universidad—, por lo menos se mantuvo igual o disminuyó, como reporta la literatura que ha pasado en los estudiantes universitarios.

Investigaciones sobre el tema se han llevado a cabo en otros países, en donde la condición física y los hábitos alimentarios de los estudiantes de Educación Física ha sido motivo de indagación y se ha reportado en el estudio de Almagia, Lizana, Rodríguez, Ivanovic \& Binvignat (2009) en el que evaluaron a 50 estudiantes de primer año de la carrera de Educación Física de la Pontificia Universidad Católica de Valparaíso, los componentes antropométricos y la condición física, encontrando que la masa grasa de hombres y mujeres eran más bajos y la masa muscular más alta en comparación con otros estudiantes, además de existir una relación entre la masa grasa y las pruebas de velocidad, resistencia y fuerza en miembros superiores en los hombres. Para las mujeres existió una correlación positiva entre la masa muscular y la prueba de velocidad. Sin embargo, los investigadores concluyeron que "las variables antropométricas del grupo universitario evaluado, no se correlacionan de manera importante con el rendimiento físico, ya que el nivel de entrenamiento es bajo".

Durán, Valdés, Godoy y Herrera (2014) evaluaron a 420 estudiantes de Pedagogía en Educación Física de la Universidad Autónoma de Chile, desde la cohorte de 2009 hasta la cohorte del año 2013, por medio de la "encuesta sobre hábitos alimentarios" y las pruebas descritas en el sistema de medición de calidad de la educación de Educación Física. Entre los hallazgos encontraron un mejor rendimiento físico de las cohortes 2010 y 2012, debido a la mayor carga académica en asignaturas prácticas; los autores concluyen que los estudiantes "presentan malos hábitos alimentarios durante toda la carrera universitaria, gran parte de ellos presentan sobrepeso u obesidad, mientras que su condición física disminuye de manera irregular a medida que avanzan los estudios".

Ahora bien, en nuestro contexto hay pocos estudios que determinen un perfil en la condición física de los estudiantes universitarios y que hayan realizado seguimiento en el tiempo de esta. Por ello, esta investigación tiene por objetivo determinar los cambios de los estudiantes de las seis cohortes con corte a 2017-I en composición corporal, fuerza, flexibilidad, velocidad, agilidad y consumo de oxígeno que ingresaron a la universidad entre el 2013-II y el 2016-II. 


\section{Metodología}

Diseño metodológico: este estudio es de corte cuantitativo, con una cohorte de seguimiento prospectivo desde el 2013-I hasta el 2016-II, con corte a 2017-I, de alcance descriptivo.

Participantes: se tomó como población de estudio a los estudiantes de la Facultad de Cultura Física, Deporte y Recreación que ingresaron desde el primer semestre de 2013 y se han mantenido hasta el segundo semestre de 2016. Los criterios de inclusión que se tuvieron en cuenta establecían que podrían participar hombres y mujeres estudiantes de la Facultad de Cultura Física, Deporte y Recreación de la Universidad Santo Tomás, que hubiesen permanecido activos (que se han matriculado ininterrumpidamente todos los semestres) desde el ingreso hasta la fecha del corte que es 2017-I. Los criterios de exclusión no permitían la participación de estudiantes que hubieran presentado lesiones tales como desgarros de la musculatura isquiotibial y los cuádriceps, o cualquier otro que le impida desarrollar todas las pruebas físicas, así mismo, no podían haber padecido fracturas, cirugías o dolor en la columna vertebral en los últimos 3 meses, lo que les impediría realizar las pruebas post de condición física. Tampoco fueron admitidos estudiantes que no hubieran permanecido activos de manera constante en el periodo de seguimiento.

Tamaño muestral: de acuerdo con los criterios de inclusión (hombres y mujeres estudiantes de la Facultad de Cultura Física, Deporte y Recreación de la Universidad Santo Tomás, que hayan permanecido activos como estudiantes desde el ingreso hasta la fecha del corte, es decir, hasta 2017-I. De este modo se obtuvieron un total de 470 sujetos para evaluar. En los meses de agosto y septiembre se realizó la convocatoria para valoración de la población. Se logró evaluar a los 132 estudiantes esperados de acuerdo con la fórmula estimada para esto (ver Tabla 1).

Tabla 1. Tamaño muestral

\begin{tabular}{|l|c|}
\hline Total de la población $(\mathrm{N})$ & 470 \\
\hline (Si la población es infinita, dejar la casilla en blanco) & \\
\hline Nivel de confianza o seguridad $(1-\alpha)$ & $95 \%$ \\
\hline
\end{tabular}

Continúa tabla... 


\begin{tabular}{|l|r|}
\hline Precisión (d) & $3.5 \%$ \\
\hline Proporción (valor aproximado del parámetro que queremos medir) & $5 \%$ \\
\hline (Si no tenemos dicha información p=0.5 que maximiza el tamaño muestral) & \\
\hline Tamaño muestral (n) & 113 \\
\hline El tamaño muestral ajustado a pérdidas & \\
\hline Proporción esperada de pérdidas (R) & $15 \%$ \\
\hline Muestra ajustada a las pérdidas & 132 \\
\hline
\end{tabular}

Fuente: elaboración propia, 2017.

Métodos y procedimientos: las pruebas escogidas para su aplicación ya están validadas a nivel internacional y se utilizarán equipos que hacen parte del Laboratorio de Fisiología de la Facultad de Cultura Física, Deporte y Recreación (ver Tabla 2).

Tabla 2. Pruebas de condición física

\begin{tabular}{ll}
\hline \multicolumn{1}{c}{ Componente } & \multicolumn{1}{c}{ Pruebas } \\
\hline Fuerza (espalda y mano) & Dinamometría \\
Flexibilidad & Test de Wells \\
\multirow{2}{*}{ Agilidad } & Prueba de obstáculos \\
& Adaptación al móvil \\
\hline
\end{tabular}

Fuente: Modelo de ingreso, 2013.

Las pruebas físicas fueron aplicadas a los estudiantes para el ingreso a la Facultad de Cultura Física, Deporte y Recreación, por lo que ya se tienen almacenados los datos de preingreso y se volverán a aplicar en el primer semestre del 2017 (datos post-ingreso). La hipótesis de investigación que se maneja es que la condición física de los estudiantes debió mejorar durante su permanencia en la Facultad.

Consideraciones éticas: la investigación se desarrolló de acuerdo con la declaración de Helsinki, la Resolución No. 008430 de 1993 del Ministerio de Salud colombiano. Esta investigación se clasificó como de riesgo mínimo, 
según lo establecido en el artículo 11, numeral b de la resolución 008430 del Ministerio de Salud. Se respetó la confidencialidad de la información recopilada tanto en medio físico como electrónico.

Plan de análisis: para los datos cuantitativos se obtuvo la media (siendo esta importante si los datos son paramétricos), partiendo de lo anterior se realizará un estudio bivariado. La información se almacenará en una base de datos para analizarla a través del programa sPss versión 21.

\section{Resultados}

De los 132 estudiantes a quienes se les tomó la evaluación antes iniciar la universidad se encontró que el $81.8 \%$ fueron hombres y $18.9 \%$ restante fueron mujeres. La media de duración de la prueba — 30 metros lanzadosque evalúa velocidad fue de 4.8984 segundos $\left(\mathrm{DS}_{ \pm} \pm .02\right)$, se evaluó agilidad teniendo como media 34,797 segundos (DSะ6.44), así como la adaptación al móvil la media que correspondió a 24.224 segundos (DS \pm 8.73$)$ (ver Tabla 3).

En la evaluación de fuerza de miembros inferiores se encontró que solo se evaluó a 56 aspirantes, razón por la cual se decidió no valorarla en la prueba post. En fuerza de mano se encontró que la media es de $32.6 \mathrm{kgf}(\mathrm{DS} \pm 9.44)$ y la fuerza de espalda la media es de 102.46 Kgf (DS \pm 30.92). Se evalúo la flexibilidad con una media de $7.64 \mathrm{~cm}\left(\mathrm{DS}_{ \pm} 8\right)$ y, por último, se evaluó el $\mathrm{VO}_{2}$ encontrando una media de $43.24 \mathrm{ml} / \mathrm{kg} / \mathrm{min}$ (ver Tabla 3 ).

Tabla 3. Pruebas antes de iniciar la universidad

\begin{tabular}{|c|c|c|c|c|c|c|c|c|c|c|}
\hline & 总 & 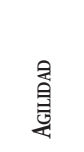 & 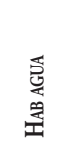 & 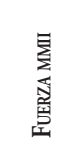 & $\begin{array}{l}\text { 竞 } \\
\text { 离 } \\
\text { 意 }\end{array}$ & 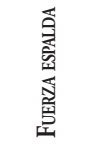 & 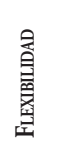 & $\underbrace{-}$ & 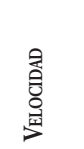 & 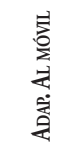 \\
\hline Válidos & 132 & 131 & 75 & 56 & 129 & 130 & 128 & 125 & 102 & 109 \\
\hline Perdidos & 0 & 1 & 57 & 76 & 3 & 2 & 4 & 7 & 30 & 23 \\
\hline Media & 1,19 & 34,797 & 57,109 & 40,907 & 32,636 & 102,462 & 7,641 & 43,243328 & 4,8984 & 24,2248 \\
\hline Mediana & 1,00 & 34,350 & 51,360 & 39,600 & 32,000 & 100,500 & 9,000 & 45,000000 & 3,7000 & 22,1000 \\
\hline
\end{tabular}




\begin{tabular}{|c|c|c|c|c|c|c|c|c|c|c|c|}
\hline & & 趈 & 嬊 & 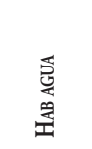 & 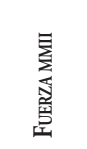 & 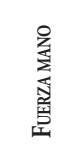 & 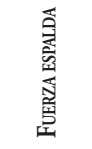 & 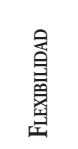 & $0^{-}$ & 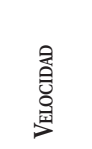 & 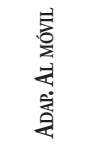 \\
\hline Desv. típ. & & 393 & 6,4497 & 23,1160 & 7,2323 & 9,4494 & 30,9213 & 8,0044 & 10,3577970 & 5,02686 & 8,73905 \\
\hline Varianza & &, 155 & 41,598 & 534,349 & 52,306 & 89,292 & 956,126 & 64,071 & 107,284 & 25,269 & 76,371 \\
\hline Rango & & 1 & 40,0 & 121,6 & 32,0 & 66,0 & 173,0 & 48,0 & 76,2000 & 29,00 & 53,20 \\
\hline Suma & & 157 & 4558,4 & 4283,2 & 2290,8 & 4210,0 & 13320,0 & 978,0 & 5405,4160 & 499,64 & 2640,50 \\
\hline \multirow{3}{*}{ Percentiles } & 25 & 1,00 & 30,400 & 42,000 & 35,700 & 26,000 & 80,750 & 2,000 & 39,112000 & 3,4375 & 19,0000 \\
\hline & 50 & 1,00 & 34,350 & 51,360 & 39,600 & 32,000 & 100,500 & 9,000 & 45,000000 & 3,7000 & 22,1000 \\
\hline & 75 & 1,00 & 38,400 & 63,000 & 45,525 & 39,000 & 124,250 & 13,000 & 47,900000 & 4,0250 & 25,8500 \\
\hline
\end{tabular}

Fuente: elaboración propia, 2017.

Al buscar las diferencias por sexo se halló que los hombres son más agiles que las mujeres (ver Figura 1).

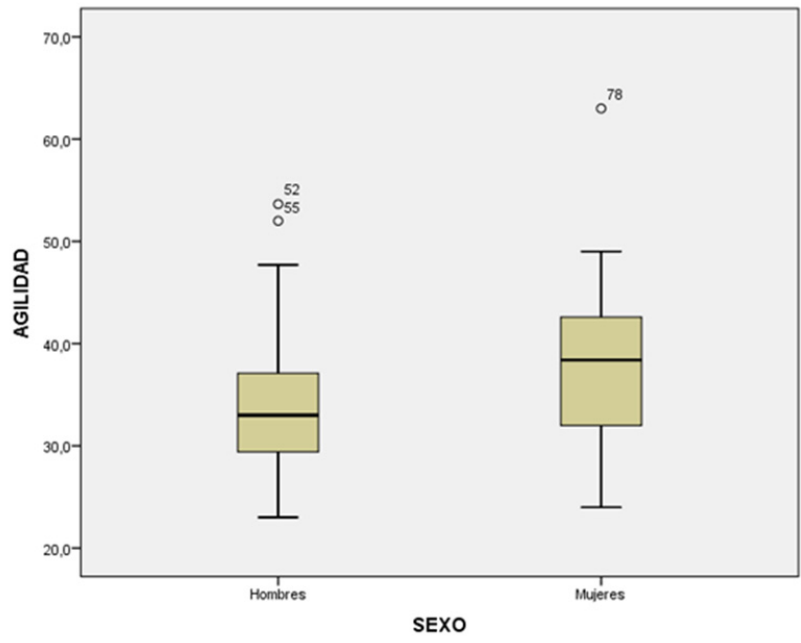

Figura 1. Agilidad antes de iniciar la universidad por sexo.

Fuente: elaboración propia, 2017.

En fuerza de mano se encontró por sexo que los hombres tienen mayor fuerza que las mujeres (ver Figura 2). 


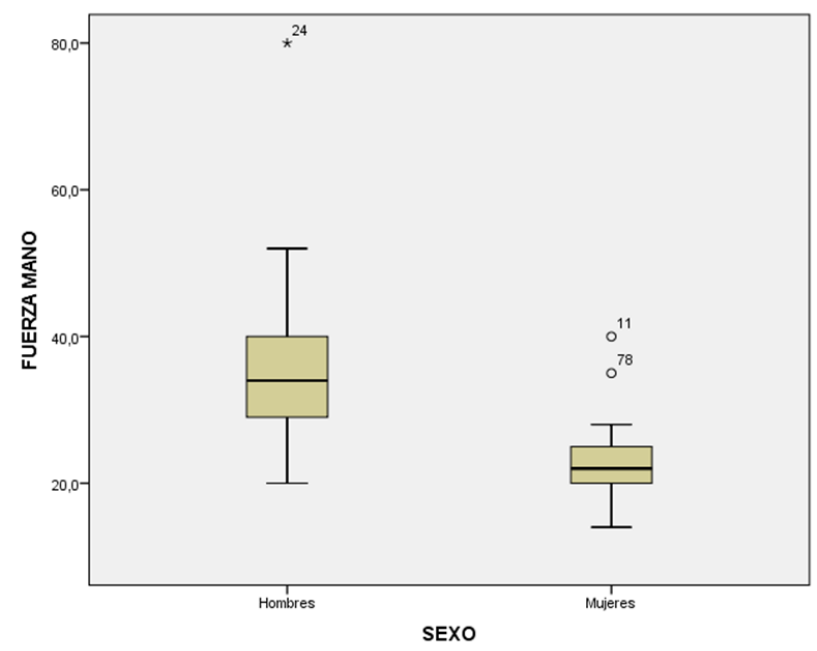

Figura 2. Fuerza de mano antes de iniciar la universidad por sexo.

Fuente: elaboración propia, 2017.

En fuerza de espalda se encontró por sexo que los hombres tienen mayor fuerza que las mujeres (ver Figura 3).

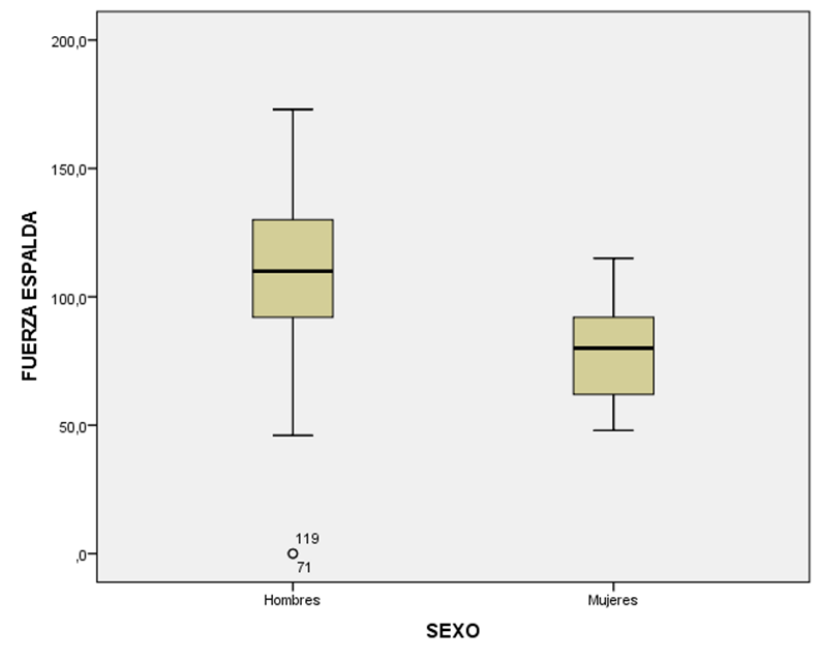

Figura 3. Fuerza de espalda antes de iniciar la universidad por sexo.

Fuente: elaboración propia, 2017. 
En el consumo de oxígeno $\mathrm{VO}_{2}$, se encontró que los hombres están ligeramente por encima de las mujeres, aunque hay valores muy bajos para aquellos que no alcanzaron el estadio 1 de la prueba de Leger (ver Figura 4).

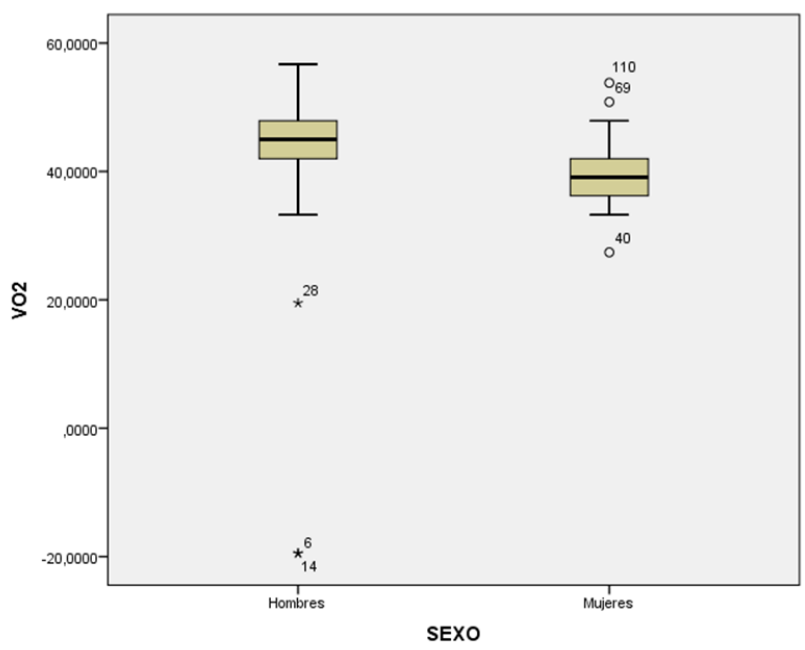

Figura 4. Consumo de oxígeno antes de iniciar la universidad por sexo.

Fuente: elaboración propia, 2017.

En los resultados de las pruebas realizadas en el corte 2017-1, las cuales hacen referencia a los resultados durante la estancia en la universidad de los estudiantes, se encontró una media de duración de 4.1434 segundos $(\mathrm{DS} \pm 0.47$ ) para la prueba 30 metros lanzados, que evalúa velocidad, estos valores fueron superiores a los que se encontraron en la evaluación pre, concluyendo que la velocidad aumentó. Otros valores que mejoraron fueron los tiempos de adaps tación al móvil, teniendo una media de 19.833 segundos (DS \pm 5.88 ), y los valores generales de fuerza, encontrando la media en fuerza de mano de 41.38 $\mathrm{Kgf}(\mathrm{DS} \pm 11.37)$ y la fuerza de espalda la media en $105.618 \mathrm{Kgf}\left(\mathrm{DS}_{ \pm}\right.$8.61) (ver tabla 4). 
Tabla 4. Pruebas durante su instancia en la universidad

\begin{tabular}{|c|c|c|c|c|c|c|c|}
\hline & 离 & 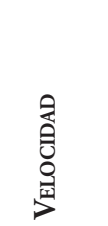 & $\begin{array}{l}\text { 令 } \\
\text { 畺 } \\
\text { 兽 }\end{array}$ & 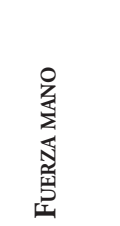 & 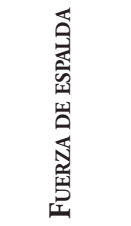 & 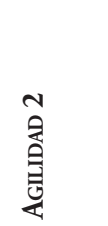 & 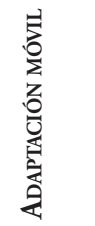 \\
\hline \multirow{2}{*}{$\begin{array}{l}\text { Válidos } \\
\text { Perdidos }\end{array}$} & 132 & 59 & 127 & 127 & 110 & 108 & 108 \\
\hline & 0 & 73 & 5 & 5 & 22 & 24 & 24 \\
\hline Media & 1,19 & 4,1434 & 2,709 & 41,387 & 105,618 & 42,137 & 19,833 \\
\hline Mediana & 1,00 & 4,0100 & 4,000 & 42,000 & 108,000 & 41,400 & 18,330 \\
\hline Desv. típ. & ,393 & ,47694 & 9,1340 & 11,3716 & 31,6471 & 8,6130 & 5,8832 \\
\hline Varianza & ,155 & ,227 & 83,430 & 129,314 & 1001,541 & 74,185 & 34,612 \\
\hline Rango & 1 & 2,07 & 44,0 & 79,0 & 130,0 & 56,9 & 38,1 \\
\hline Suma & 157 & 244,46 & 344,0 & 5256,2 & 11618,0 & 4550,8 & 2141,9 \\
\hline
\end{tabular}

Fuente: elaboración propia, 2017.

Pero no en todas las evaluaciones se encontró una mejoría, ya que en la evaluación de agilidad la media fue de 42.137 segundos $(D S \pm 8.6)$ y en la de flexibilidad la media fue de $2.709 \mathrm{~cm}\left(\mathrm{DS}_{ \pm} \pm .13\right)$, valores inferiores a los de preingreso a la universidad (ver tabla 4). La prueba de $\mathrm{VO}_{2}$ no se pudo realizar por los tiempos de los estudiantes, así que no fue posible realizar una comparación con respecto a este dato en el corte 2017-1.

$\mathrm{Al}$ hacer la comparación por sexo, se encontró en velocidad que los hombres siguen siendo más ágiles que las mujeres (Figura 5).

En flexibilidad, los resultados en mujeres son mejores y la desviación estándar es menor que en los hombres (ver Figura 6). 


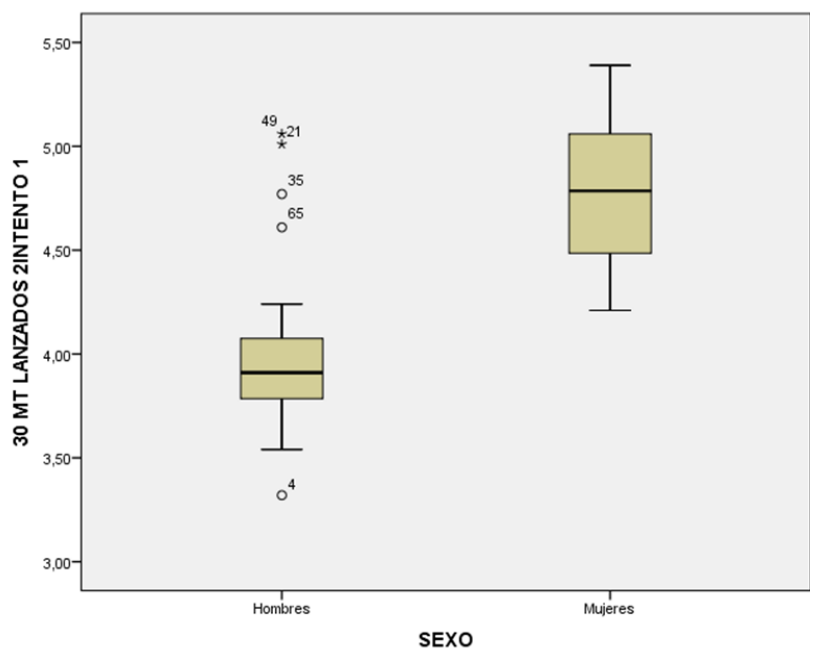

Figura 5. Velocidad por sexo en pruebas durante la universidad.

Fuente: elaboración propia, 2017.

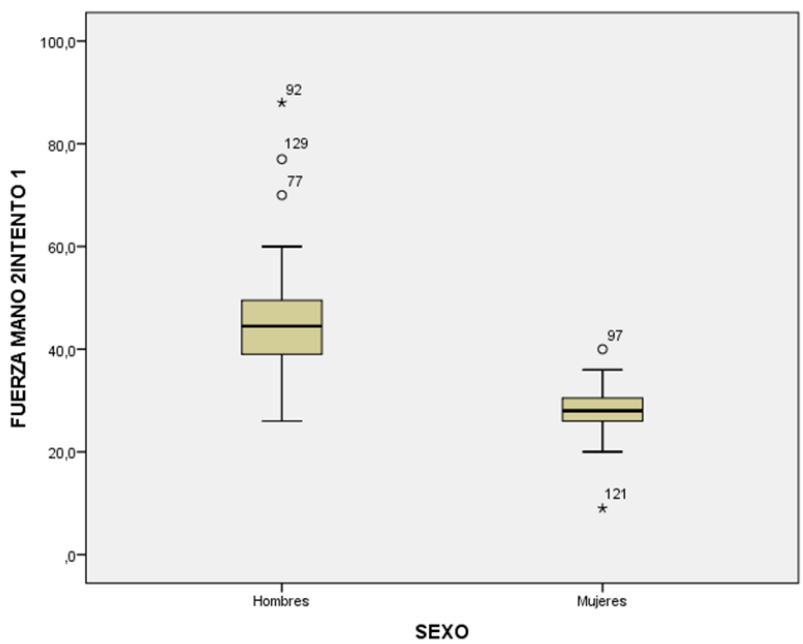

Figura 6. Flexibilidad por sexo en pruebas durante la universidad.

Fuente: elaboración propia, 2017.

En fuerza muscular de mano los hombres presentaron mejores resultados que las mujeres, aunque en este grupo la desviación estándar es menor (ver Figura 7). 


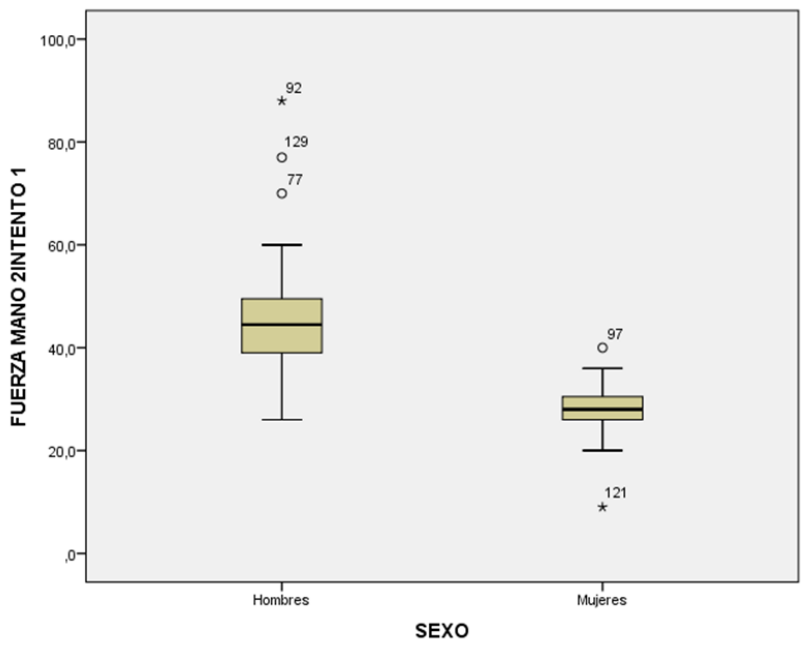

Figura 7. Fuerza de mano por sexo en pruebas durante la universidad. Fuente: elaboración propia, 2017.

En fuerza muscular de espalda los hombres presentaron mejores resultados que las mujeres, aunque en este grupo la desviación estándar es menor (Figura 8).

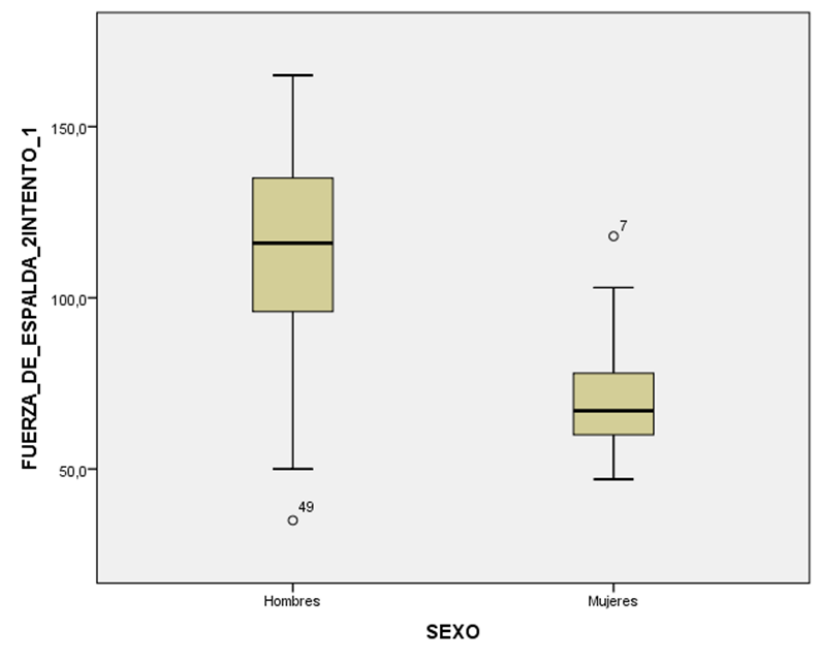

Figura 8. Fuerza de espalda por sexo en pruebas durante la universidad. Fuente: elaboración propia, 2017. 
Al hacer la comparación con las diferentes cohortes, no se encuentra relación estadísticamente significativa entre los resultados obtenidos en cada una de aquellas y las pruebas realizadas de la condición física (ver tabla 5).

Tabla 5. Comparación de resultados durante la instancia de la universidad

\begin{tabular}{|c|c|c|c|c|c|c|c|}
\hline & & \multirow{2}{*}{$\mathbf{N}$} & \multirow{2}{*}{ Media } & \multirow{2}{*}{$\begin{array}{l}\text { Desviación } \\
\text { típica }\end{array}$} & \multirow{2}{*}{$\begin{array}{l}\text { Error } \\
\text { típico }\end{array}$} & \multicolumn{2}{|c|}{$\begin{array}{l}\text { Intervalo de confianza } \\
\text { para la media al } 95 \%\end{array}$} \\
\hline & & & & & & $\begin{array}{l}\text { Límite } \\
\text { inferior }\end{array}$ & $\begin{array}{l}\text { Límite } \\
\text { superior }\end{array}$ \\
\hline \multirow{7}{*}{$\begin{array}{l}30 \mathrm{MT} \\
\text { LANZADOS } 2 \\
\text { INTENTO I }\end{array}$} & 2013-1 & 36 & 4,1036 & ,48572 & 08095 & 3,9393 & 4,2680 \\
\hline & $2013-2$ & 2 & 5,1250 & ,37477 & ,26500 & 1,7579 & 8,4921 \\
\hline & 2014-1 & 2 & 4,5900 & ,66468 & ,47000 & $-1,3819$ & 10,5619 \\
\hline & $2014-2$ & 8 & 4,1588 & ,28578 & , 10104 & 3,9198 & 4,3977 \\
\hline & $2015-1$ & 7 & 4,0200 & ,43159 & , 16312 & 3,6208 & 4,4192 \\
\hline & $2015-2$ & 4 & 3,9725 & ,13672 & ,06836 & 3,7550 & 4,1900 \\
\hline & Total & 59 & 4,1434 & ,47694 & ,06209 & 4,0191 & 4,2677 \\
\hline \multirow{7}{*}{$\begin{array}{l}\text { WELLS } 2 \\
\text { INTENTO I }\end{array}$} & 2013-1 & 37 & 2,919 & 7,8045 & 1,2830 &, 317 & 5,521 \\
\hline & $2013-2$ & 3 & 3,000 & 9,0000 & 5,1962 & $-19,357$ & 25,357 \\
\hline & 2014-1 & 12 & 3,417 & 9,4143 & 2,7177 & $-2,565$ & 9,398 \\
\hline & $2014-2$ & 14 & 3,714 & 9,5951 & 2,5644 & $-1,826$ & 9,254 \\
\hline & $2015-1$ & 42 & 2,214 & 10,0815 & 1,5556 &,- 927 & 5,356 \\
\hline & $2015-2$ & 19 & 2,158 & 9,9458 & 2,2817 & $-2,636$ & 6,952 \\
\hline & Total & 127 & 2,709 & 9,1340 &, 8105 & 1,105 & 4,313 \\
\hline \multirow{7}{*}{$\begin{array}{l}\text { FUERZA } \\
\text { MANO } 2 \\
\text { INTENTO I }\end{array}$} & 2013-1 & 35 & 38,943 & 9,6037 & 1,6233 & 35,644 & 42,242 \\
\hline & $2013-2$ & 3 & 30,000 & 9,5394 & 5,5076 & 6,303 & 53,697 \\
\hline & 2014-1 & 12 & 45,333 & 10,5169 & 3,0360 & 38,651 & 52,015 \\
\hline & $2014-2$ & 17 & 43,276 & 8,8522 & 2,1470 & 38,725 & 47,828 \\
\hline & 2015-1 & 41 & 41,780 & 12,3602 & 1,9303 & 37,879 & 45,682 \\
\hline & $2015-2$ & 19 & 42,658 & 13,9673 & 3,2043 & 35,926 & 49,390 \\
\hline & Total & 127 & 41,387 & 11,3716 & 1,0091 & 39,390 & 43,384 \\
\hline
\end{tabular}




\begin{tabular}{|c|c|c|c|c|c|c|c|}
\hline & & \multirow{2}{*}{$\mathbf{N}$} & \multirow{2}{*}{ Media } & \multirow{2}{*}{$\begin{array}{l}\text { Desviación } \\
\text { típica }\end{array}$} & \multirow{2}{*}{$\begin{array}{l}\text { Error } \\
\text { típico }\end{array}$} & \multicolumn{2}{|c|}{$\begin{array}{l}\text { Intervalo de confianza } \\
\text { para la media al } 95 \%\end{array}$} \\
\hline & & & & & & $\begin{array}{l}\text { Límite } \\
\text { inferior }\end{array}$ & $\begin{array}{l}\text { Límite } \\
\text { superior }\end{array}$ \\
\hline \multirow{7}{*}{$\begin{array}{l}\text { FUERZA DE } \\
\text { ESPALDA } 2 \\
\text { INTENTO I }\end{array}$} & 2013-1 & 36 & 107,389 & 33,4068 & 5,5678 & 96,086 & 118,692 \\
\hline & $2013-2$ & 3 & 86,333 & 25,1064 & 14,4952 & 23,965 & 148,701 \\
\hline & 2014-1 & 8 & 116,625 & 35,8447 & 12,6730 & 86,658 & 146,592 \\
\hline & $2014-2$ & 15 & 110,533 & 31,9774 & 8,2565 & 92,825 & 128,242 \\
\hline & $2015-1$ & 31 & 100,613 & 28,3804 & 5,0973 & 90,203 & 111,023 \\
\hline & $2015-2$ & 17 & 104,882 & 33,5669 & 8,1412 & 87,624 & 122,141 \\
\hline & Total & 110 & 105,618 & 31,6471 & 3,0174 & 99,638 & 111,599 \\
\hline \multirow{7}{*}{$\begin{array}{l}\text { AGILIDAD } 2 \\
\text { INTENTO I }\end{array}$} & 2013-1 & 37 & 43,640 & 9,6001 & 1,5782 & 40,439 & 46,841 \\
\hline & $2013-2$ & 2 & 46,150 & 9,4187 & 6,6600 & $-38,473$ & 130,773 \\
\hline & 2014-1 & 9 & 45,147 & 7,9087 & 2,6362 & 39,067 & 51,226 \\
\hline & $2014-2$ & 17 & 37,728 & 5,7321 & 1,3902 & 34,781 & 40,675 \\
\hline & $2015-1$ & 36 & 41,374 & 8,7011 & 1,4502 & 38,430 & 44,318 \\
\hline & $2015-2$ & 7 & 43,804 & 6,9184 & 2,6149 & 37,406 & 50,203 \\
\hline & Total & 108 & 42,137 & 8,6130 & ,8288 & 40,494 & 43,780 \\
\hline \multirow{7}{*}{$\begin{array}{l}\text { ADAPTACIÓN } \\
\text { BALÓN } 2 \\
\text { INTENTO I }\end{array}$} & 2013-1 & 37 & 21,097 & 6,2949 & 1,0349 & 18,998 & 23,196 \\
\hline & $2013-2$ & 1 & 45,200 & . & . & . & . \\
\hline & 2014-1 & 10 & 17,119 & 2,4213 & ,7657 & 15,387 & 18,851 \\
\hline & $2014-2$ & 7 & 18,137 & 2,9114 & 1,1004 & 15,445 & 20,830 \\
\hline & $2015-1$ & 35 & 19,199 & 5,7764 & ,9764 & 17,215 & 21,184 \\
\hline & $2015-2$ & 18 & 19,222 & 3,6110 &, 8511 & 17,426 & 21,018 \\
\hline & Total & 108 & 19,833 & 5,8832 &, 5661 & 18,710 & 20,955 \\
\hline
\end{tabular}

Fuente: elaboración propia, 2017.

Por último, se hizo una la prueba t para variables pareadas y se halló una relación estadísticamente significativa entre la flexibilidad y la fuerza de espalda; en flexibilidad con la prueba pre y post se obtuvo una correlación de $0.617(\mathrm{p}=0.000)$ y en fuerza de espalda de $0.799(\mathrm{p}=0.000)$. En las pruebas 
de fuerza de mano pre y post la correlación fue de 0.366 ( $\mathrm{p}=0.031)$, agilidad (prueba de obstáculos) la correlación fue de $0.210(\mathrm{p}=0.212)$ y adaptación al móvil de 0.288 ( $\mathrm{p}=0.084)$.

Un comportamiento diferente se encontró con la prueba de Wilcoxon, pues en algunas de las variables de las pruebas físicas se presentaron diferencias estadísticamente significativas, como se observa en la Tabla 6.

Tabla 6. Prueba de Wilconxon para variables no paramétricas

\begin{tabular}{lc}
\hline \multicolumn{1}{c}{ Variable } & Valor de p \\
\hline Flexibilidad & $0.000^{*}$ \\
Fuerza de mano & $0.000^{*}$ \\
Fuerza de espalda & $0.000^{*}$ \\
Agilidad (prueba de obstáculos) & $0.000^{*}$ \\
Agilidad (adaptación al móvil) & $0.000^{*}$ \\
\hline Nota. ${ }^{*}<0.05$ &
\end{tabular}

Fuente: elaboración propia, 2017.

\section{Discusión}

Las universidades del mundo están recibiendo anualmente una gran cantidad de estudiantes; en Colombia, de acuerdo con el sistema nacional de instituciones de educación superior (Sistema Nacional de Información de la Educación Superior, 2015), en el 2015 se matricularon para el primer curso universitario 785.878 estudiantes, que ingresaron a los diferentes programas de pregrado y posgrado en espera de una formación profesional integral. Cuando se piensa en el término "integralidad" son muchos los aspectos que entran en juego, las universidades deben velar porque se brinde no solo un desarrollo a nivel cognitivo, sino también en lo que respecta a la condición física y a la generación de hábitos saludables. Para ello establecen diferentes estrategias como, por ejemplo, ofertar espacios académicos en los cuales esté presente la actividad física y el deporte. Ahora bien, para las universidades que 
ofertan programas específicos, enfocados a la educación física, la cultura física, el deporte y la recreación, está preocupación se torna aún mayor, pues los estudiantes no solo deben poseer una condición física adecuada, sino que además deben transmitir y desarrollar estilos de vida saludables. Los resultados de este estudio evidencian que existen cualidades del movimiento que no se trabajan constantemente en el desarrollo de la carrera, lo que generó una disminución global al finalizar los estudios, por otro lado, algunas cualidades que parece que sí se trabajaron presentaron mejoría.

$\mathrm{Al}$ revisar lo publicado en la literatura mundial frente a los cambios reportados en flexibilidad, se encontró un estudio en Colombia en el que se entrenó a brigadistas universitarios en 12 semanas y se encontró que esta cualidad mejoró en $1 \mathrm{~cm}$, aunque los cambios no fueros estadísticamente significativos como en la presente investigación, en la que esta cualidad no aumentó sino que disminuyó, por lo que la posibilidad de obtener mejoría en la flexibilidad depende de un programa o de una serie de intervenciones que tengan en cuenta la evaluación, prescripción de ejercicio, recomendaciones y seguimiento (León-Rodríguez, \& Cañón-Betancourt, 2013), por lo que se podría inferir que esta cualidad no se trabaja constantemente durante la instancia en la universidad de la población y por eso desmejoró (Arregui \& Martínez, 2001).

En un estudio realizado por García-Soidán \& Alonso (2011), se encontraron resultados en fuerza manual por encima de los nuestros, tanto en los valores de hombres como mujeres y en flexibilidad, los resultados fueron inferiores a la baremación con calificación de normal o promedio, en las tres universidades gallegas estudiadas, tanto en mujeres como en hombres (con un valor de $\mathrm{p}=0,001$ en ambos casos), con un 63\% del total de la muestra, presentando valores inferiores a los normales para su edad y género. Esto evidencia que en el periodo universitario no solo se trabaja poco, sino que también el cuerpo adopta durante muchas horas de estudio las mismas posturas, lo que podría justificar esa pérdida importante de la flexibilidad anterior del tronco, conllevando a problemas articulares u osteomusculares degenerativos importantes en la etapa adulta (Marín, Naclerio, Viejo \& Forte, 2007; García-Soidán \& Alonso, 2011). 
Entre las limitaciones que presenta el estudio se encuentra el no reconocimiento de otras variables que pueden afectar la condición física, como lo es pertenecer a alguna selección deportiva o padecer de alguna patología concomitante que pueda afectar las condiciones de salud (Oviedo et al., 2008).

Por otro lado, la disminución de las condiciones físicas (que se observaron en algunas variables) en el ámbito universitario, se ha asociado a mayor riesgo de sufrir enfermedades crónicas como las de origen cardiometabólico y cardiovascular, por lo que es necesario aunar esfuerzos para reforzar la condición física durante la instancia universitaria (Dadelo \& Tamoðauskas, 2005; Maldonado et al., 2013), así mismo, se han asociado los beneficios de la actividad física para la salud y el bienestar, pero las características de los entornos universitarios limitan la capacidad de los estudiantes para realizar actividad física o participar en actividades beneficiosas para su salud (Lovasi et al., 2013).

Para próximos estudios, se sugiere relacionar la condición física con el rendimiento académico, ya que se ha encontrado evidencia sobre la relación de estos en un estudio realizado por Van Dusen et al., (2011) quienes mencionan entre sus resultados que la aptitud física se relaciona de manera importante y significativa con el rendimiento académico. La aptitud cardiovascular mostró una asociación dosis-respuesta con el rendimiento académico independiente de otras variables sociodemográficas y de aptitud. La asociación parece alcanzar su pico en la escuela secundaria, concluyendo que, al aumentar el tiempo de educación física, en especial en entrenamiento de la aptitud cardiovascular, se pueden mejorar los resultados académicos en la escuela (Van Dusen et al., 2011).

\section{Conclusiones}

Los resultados de las pruebas realizadas en el corte 2017-1, las cuales hacen referencia a los resultados durante la estancia en la universidad de los estudiantes, muestran una mejora en las diferentes cortes en la prueba 30 metros lanzados, adaptación al móvil (agilidad) y los valores generales de fuerza (espalda y mano).

Pero no en todas las evaluaciones se encontró una mejoría, ya que en la evaluación de agilidad (obstáculos) y flexibilidad disminuyeron. La prueba de $\mathrm{VO}_{2}$ no se pudo hacer por los tiempos de los estudiantes, así que no fue posible 
realizar una comparación con respecto a este en el corte 2017-1. Se encontró, adicionalmente, que al hacer la comparación con las diferentes cohortes no se da una relación estadísticamente significativa entre los resultados obtenidos en cada una de las cohortes y las pruebas realizadas de la condición física.

\section{Referencias}

Almagia, A., Lizana, P., Rodríguez, F., Ivanovic, D., \& Binvignat, O. (2009). Variables antropométricas y rendimiento físico en estudiantes universitarios de educación física. International journal of morphology, 27(4), 971-975.

Arregui, J., \& Martínez, V. (2001). Estado actual de las investigaciones sobre la flexibilidad en la adolescencia. Revista internacional de medicina y ciencias de la actividad fisica y el deporte, 1(2), 127-135.

Córdoba, L. (2013). Modelo de ingreso. Ediciones USTA.

Dadelo, S., \& Tamoðauskas, P. (2005). Investigation and assessment of College students' physical development, physical fitness, and functional capacity. Acta Academiae Olympiquae Estonia, 13(2), 55-66.

Durán, A., Valdés, B., Godoy, C. \& Herrera, V. (2014). Hábitos alimentarios y condición física en estudiantes de pedagogía en educación física. Revista chilena de nutrición, 41(3), 251-259.

García-Soidán, J., \& Alonso, D. (2011). Valoración de la condición física saludable en universitarios gallegos. Revista internacional de medicina y ciencias de la actividad fisica y del deporte, 11(44), 781-790.

León, A, \& Zea, R. (2010). Hábitos y factores de riesgo cardiovascular en estudiantes de primer semestre de la Universidad Santo Tomás. Hallazgos: Revista de Investigaciones, 7(13), 181-191

León-Rodríguez, J., \& Cañón-Betancourt, L. (2013). Efecto del ejercicio prescrito no controlado sobre el perfil de condición física en brigadistas universitarios. Revista de salud pública, 15(2), 216-226.

Lovasi, G., Schwartz-Soicher, O., Quinn, J., Berger, D., Neckerman, K., Jaslow, R., Lee, K., \& Rundle, A. (2013). Neighborhood safety and green space as predictors of obesity among preschool children from low-income families in New York City. Preventive medicine, 57(3), 189-193.

Maldonado, J., Carranza, C., Ortiz, M., Gómez, C., \& Cortés-Gallegos, N. (2013). Prevalencia de factores de riesgo cardiometabólico en estudiantes universitarios de la región centro-occidente, en la Universidad Michoacana de San Nicolás de Hidalgo, México. Revista mexicana de cardiología, 24(2), 76-86.

Marín, P., Naclerio, F., Viejo, D., \& Forte, D. (2007). Efectos de diferentes protocolos de entrenamiento de fuerza sobre la fuerza máxima, la velocidad, la saltabilidad y el equilibrio en estudiantes universitarios. Revista Kronos, 6(12), 12-21. 
Oviedo, G., Morón de Salim, A., Santos, I., Sequera, S., Soufrontt, G., Suárez, P., \& Arpaia, A. (2008). Factores de riesgo de enfermedades crónicas no ransmisibles en estudiantes de la carrera de Medicina: Universidad de Carabobo, Venezuela. Año 2006. Nutrición Hospitalaria, 23(3), 288-293.

Sistema Nacional de Información de la Educación Superior (SNIES) (2015). Población estudiantil: matriculados en primer curso. http://www.mineducacion.gov.co/sistemasdeinformacion/1735/w3-article-212400.html

Van Dusen, D., Kelder, S., Kohl III, H., Ranjit, N., \& Perry, C. (2011). Associations of physical fitness and academic performance among schoolchildren. Journal of school health, 81(12), 733-740. 